\title{
ПРОГНОСТИЧЕСКИЕ КРИТЕРИИ РАЗВИТИЯ ТЯЖЕЛОГО ПОСТЭМБОЛИЗАЦИОННОГО СИНДРОМА У ПАЦИЕНТОК С МИОМОЙ МАТКИ
}

\author{
Э. Т. Нурмухаметова $\bowtie$, М. Е. Шляпников
}

Медицинский университет «Реавиз», Самара, Россия

В последние годы проявляется интерес к органосохраняющим методам лечения миомы матки, одним из которых является эмболизация маточных артерий (ЭМА). Среди отрицательных сторон метода - развитие тяжелого постэмболизационного синдрома (ПЭС), требующего своевременного начала адекватного лечения с целью избежания грозных осложнений, способных привести к удалению органа. Целью исследования был поиск прогностических критериев развития тяжелого ПЭС в дооперационном периоде. В исследование вошла 81 женщина с миомой матки 7-17 недель в возрасте 19-50 лет, прошедшая лечение методом ЭМА. В работе использовали антропометрические данные пациенток и показатели кожной микроциркуляции, полученные методом лазерной допплеровской флоуметрии (ЛДФ) с проведением окклюзионной пробы (ОП). На основании прогностических критериев построены модели с AUC (область под графиком ROC кривой) > 0,8. Антропометрические предикторы тяжелого ПЭС в моделях: возраст менее 38,5 лет ( $p<0,05)$; ИМТ менее 25 кг/м² ( $<$ 0,05) и показатель микроциркуляции (М) до ЭМА - менее 9,55 пф. ед. ( $p=0,001)$. Показатели микрокровотока в ОП свидетельствуют о том, что чем выше значения индекса потребления кислорода (I), внутрисосудистого сопротивления (Rc), резерва капиллярного кровотока (РКК) в моделях, тем выше риск развития тяжелой формы ПЭС ( $p<0,05)$. Низкие показатели угла альфа в ОП ( $p=0,003)$ и эндотелиальных колебаний UVLF ( $p=0,004)$ в моделях также ведут к повышенному риску развития тяжелого ПЭС. Многомерные прогностические модели позволят диагностировать развитие тяжелого ПЭС до проведения ЭМА и подготовить пациентку к определенному послеоперационному ведению и лечению.

Ключевые слова: миома матки, эмболизация маточных артерий, постэмболизационный синдром, лазерная допплеровская флоуметрия, прогностические критерии

Благодарности: к. б. н., доценту кафедры лазерных и биотехнических систем Самарского университета М. В. Комаровой за консультативную помощь в статистической обработке результатов исследования.

Вклад авторов: Э. Т. Нурмухаметова, М. Е. Шляпников - равнозначен на всех этапах работы и написания статьи.

Соблюдение этических стандартов: исследование одобрено этическим комитетом медицинского университета «Реавиз» (протокол № 2 от 14 января 2019 г.). Все пациентки подписали информированное добровольное согласие на участие в исследовании и публикацию данных.

$\triangle$ Для корреспонденции: Эльмира Тимеровна Нурмухаметова ул. Чкалова, д. 100, г. Самара, 443001; nurelm@yandex.ru

Статья получена: 31.12.2019 Статья принята к печати: 19.01.2020 Опубликована онлайн: 29.01.2020

DOI: $10.24075 /$ vrgmu.2020.006

\section{PROGNOSIS CRITERIA OF THE SEVERE POSTEMBOLIZATION SYNDROME IN PATIENTS WITH UTERINE MYOMA}

Nurmukhametova ET $\otimes$, Shlyapnikov ME

REAVIZ Medical University, Samara, Russia

Recently specialists take an interest in organ-preserving methods of uterine fibroids treatment, one of which is uterine artery embolization (UAE). One of the method's negative aspects is the severe postembolization syndrome (PES) development, requiring timely initiation of adequate treatment in order to avoid severe complications that could lead to the organ removal. The study was aimed to search for the prognostic criteria of the severe PES development during the preoperative period. The study included 81 UAE-treated women aged 19-50 with 7-17 week uterine myoma. The patients' anthropometric measurements were used, as well as the skin microcirculation data obtained by laser Doppler flowmetry together with the occlusion test. Based on prognostic criteria, models with AUC (area under ROC curve) $>0.8$ were presented. According to the models, the anthropometric predictors of the severe PES were the following: age under $38.5(p<0.05)$; BMI lower than $25 \mathrm{~kg} / \mathrm{m}^{2}(p<0.05)$, and microcirculation value (M) prior to UAE below $9.55 \mathrm{PU}(p=0.001)$. Microvascular blood flow during the occlusion test indicate that the higher the oxygen consumption index (I), intravascular resistance (Rc), capillary blood flow reserve capacity in the models, the higher the risk of the severe PES development $(p<0.05)$. Low alpha angle value obtained by the occlusion test $(p=0.003)$ as well as the UVLF value $(p=0.004)$ in the models also indicate the increased risk of severe PES. Multidimensional prognostic modelling admits to expect the severe PES development prior to UAE, which allows the doctor to prepare the woman for specific management and treatment.

Keywords: uterine fibroids, uterine artery embolization, postembolization syndrome, laser Doppler flowmetry, prognostic criteria

Acknowledgements: to MV Komarova, PhD, Associate Professor of the Samara University Department of Laser and Biotechnological Systems, for assistance in the statistical processing of the results.

Author contribution: Nurmukhametova ET, Shlyapnikov ME — the authors contributed to the study and manuscript writing equally.

Compliance with ethical standards: the study was approved by the Ethics Committee of the REAVIZ Medical University (protocol № 2 dated January 14, 2019). All patients submitted the informed consent to participation in the study and data publishing.

$\varangle$ Correspondence should be addressed: Elmira T. Nurmukhametova

Chkalova, 100, Samara, 443001; nurelm@yandex.ru

Received: 31.12.2019 Accepted: 19.01.2020 Published online: 29.01.2020

DOI: $10.24075 / \mathrm{brsmu} .2020 .006$

Миома матки занимает лидирующее положение в структуре гинекологических заболеваний, диагностируется у 20-50\% женщин репродуктивного возраста [1], а также у 18-56\% пациенток с нарушением фертильности [2]. Миома матки ухудшает качество жизни женщин активного репродуктивного возраста, их беспокоят маточные кровотечения, хроническая анемия, боли внизу живота, сдавление соседних органов и ухудшение их функций в виде нарушения работы 
мочевыделительной системы и желудочно-кишечного тракта. Основным методом лечения является удаление матки, с чем не всегда согласны пациентки и врачи [1]. В последние годы признание получают органосохраняющие методики [2]. Хирургические вмешательства, например миомэктомию лапароскопическим, лапаротомическим, влагалищным доступом, проводят под наркозом. Существует малоинвазивная эндоваскулярная методика без наркоза и разреза - эмболизация маточных артерий (ЭМА). Данный эндоваскулярный метод малоинвазивен, не требует наркоза, что привлекательно для пациенток и врачей. В результате эмболизации сосудов миомы матки нарушается питание узла, что приводит к его острой гипоксии и некрозу, ацидозу и всасыванию продуктов распада тканей в общий кровоток организма. В ответ на это у 96\% пациенток в раннем послеперационном периоде (8-36 4) развивается разной степени тяжести постэмболизационный синдром (ПЭС) [3]. Тяжесть развившегося ПЭС оценивали по шкале Ю. Э. Доброхотовой и соавт. [3].

Лечение ПЭС легкой и средней степеней тяжести ограничено назначением ненаркотических анальгетиков и нестероидных противовоспалительных средств внутрь, госпитализацией на 1-3 дня. При развитии тяжелой степени ПЭС показаны наркотические анальгетики, инфузионная терапия с антибиотиками, коррекция свертывающей системы, при необходимости - катетеризация мочевого пузыря и другие методы. При тяжелом ПЭС возникает риск развития грозных осложнений в виде метроррагии, пиометры, септических и тромбоэмболических осложнений, способных привести к органоуносящей операции [4-6]. В связи с этим актуальным остается вопрос о своевременном начале коррекционной терапии при тяжелом ПЭС, что будет способствовать более легкому течению, послужит профилактикой осложнений и создаст у пациентки благоприятное мнение о данном методе лечения. Были предложены методики снижения болевого синдрома в раннем послеоперационном периоде, так как он является ведущим симптомом. Во время проведения процедуры ЭМА перед введением эмболов предложено вводить 100 мг (2,5 мл) актовегина с целью увеличения паренхиматозного кровотока в матке, тем самым уменьшать проявления ПЭС [7]. Другие авторы рекомендуют точно рассчитывать минимальное количество порций эмболизата, что позволит снизить число осложнений, связанных с передозировкой частиц, а также стоимость процедуры за счет цены эмболов [8]. Снижает выраженность ПЭС применение венопротектерной терапии в послеоперационном периоде, особенно у больных с миомой матки, страдающих венознолимфатической недостаточностью нижних конечностей [9].

В нашем исследовании мы проанализировали анамнез пациенток, поступивших на ЭМА, и выявили, что статистически значимы такие факторы, влияющие на течение ПЭС, как возраст, ИМТ и расположение узлов. Последний служит качественной характеристикой, поэтому в прогностические модели были включены антропометрические данные (количественные). Среди методов обследования выделен метод лазерной допплеровской флоуметрии (ЛДФ) как впервые использовавшийся нами в остром периоде у пациенток с миомой матки до проведения ЭМА. В доступной литературе имеются данные об изучении кожной микроциркуляции у гинекологических больных при хронически развивающихся заболеваниях, при острых - мы не встретили [10]. Так как ПЭС - это реакция организма на процедуру ЭМА, он является системным ответом.
ЛДФ отражает изменения кожной микроциркуляции, что характеризует организм в целом [11]. Развитие именно тяжелого ПЭС сопряжено с риском развития осложнений и более выраженным влиянием на общее самочувствие пациенток, чем развитие легкого и среднего ПЭС. Целью работы было определить прогностические критерии развития тяжелого ПЭС по антропометрическим данным и особенностям кожной микроциркуляции перед процедурой ЭМА.

\section{ПАЦИЕНТЫ И МЕТОДЫ}

С 2016 по 2019 г. в ЧУЗ КБ «РЖД-Медицина» г. Самара была обследована 81 пациентка 18-50 лет с симптомной растущей миомой матки от 7 до 17 недель, с размерами узлов от 21 до 115 мм. Средний возраст наблюдаемых составил $39 \pm 6$ лет.

Критерии включения пациенток в исследование: наличие прогрессирующей миомы матки; наличие клинической симптоматики в виде менометроррагий, тянущих болей внизу живота; наличие симптома сдавления соседних органов с проявлениями в виде учащенного мочеиспускания и запоров; отсутствие в анамнезе эффекта от консервативной терапии; желание женщины сохранить матку по различным причинам, среди которых психологический комфорт, возможные повторные репродуктивные планы, большой риск оперативных вмешательств, «страх» перед наркозом и операцией.

В связи с наличием показаний всем поступившим женщинам выполнено лечение методом ЭМА. В раннем послеоперационном периоде все исследуемые были распределены на три группы по степени тяжести развившегося ПЭС: легкой степени (I группа) — 36 (44,44\%) пациенток, умеренной степени (II группа) - 30 (37,04\%), выраженной степени (III группа) - 15 (18,52\%). При выявлении множественной миомы оценивали доминантный узел. Миома матки с одиночным узлом размерами 30-110 мм была выявлена у 41 (50,6\%) пациентки, у остальных $40(49,4 \%)$ - множественная миома матки с размерами узлов от 21 до 115 мм, статистически значимых различий в группах не было $\left(p=0,705 ; \chi^{2}=0,7\right)$.

Критерии исключения из исследования по факторам, влияющим на проведение ЭМА: наличие воспалительных заболеваний малого таза; указание в анамнезе на прием агонистов гонадотропин-рилизинг гормонов или гормональных препаратов (гормональная контрацепция или заместительная терапия) менее чем в течение последних трех месяцев перед исследованием; злокачественные заболевания малого таза; аденомиоз III степени; аллергическая реакция на контрастные вещества; отрицательный тест Аллена; наличие артериовенозного шунта для проведения гемодиализа; болезнь Бюргера.

Критерии исключения из исследования по факторам, влияющим на результаты ЛДФ: наличие заболевания сердечно-сосудистой системы и атеросклеротических изменений сосудов тяжелой степени; сахарный диабет; хроническая венозная недостаточность; болезнь Рейно; облитерирующий эндоартериит; наличие травм; десрормации и инфекционные поражения ногтевых фаланг [11].

Пациенткам с субмукозными и субсерозными узлами (типы 0, 1, 6 и 7 по FIGO, 2011 г.) вместо ЭМА рекомендовали другие методы лечения: миомэктомию методом резектоскопии, лапароскопии, влагалищным доступом. Процедуру ЭМА выполняли через бедренную артерию (по методике Сельдингера) 63 пациенткам и через лучевую артерию 18 пациенткам с применением микросфер 
«Embosphere» (Biosphere Medikal; США) размерами 5001200 мкм. Выбор метода выполнения ЭМА, количество флаконов и размеров эмболов определял рентгенхирург после консультации пациентки и в ходе проведения диагностических и лечебных артериальных вмешательств.

Пациенток обследовали по общепринятым критериям: общеклиническое обследование, УзИ малого таза с цветным доплеровским картированием, консультации гинеколога, терапевта и рентгенхирурга, диагностическое выскабливание полости матки или аспирация эндометрия с гистологическим исследованием. В раннем послеоперационном периоде у пациенток развивается симптомокомплекс, называемый «постэмболизационным синдромом» (ПЭС), характеризующийся болями различной интенсивности; повышением температуры тела; увеличением СОЭ; развитием лейкоцитоза; нарушением работы сердечно-сосудистой (тахикардия), желудочно-кишечной (тошнота, рвота, парез кишечника), мочевыделительной (задержка мочеиспускания) систем; свертывающей системы (гиперфибриногенемия); появлением кровянистых выделений.

Оценку состояния системы микроциркуляции проводили до ЭМА методом ЛДФ с проведением окклюзионной пробы (ОП) как самой приближенной к процедуре ЭМА на аппарате ЛАКК-О2 («Лазма»; Россия). Исследование выполняли после акклиматизации, длящейся в течение 30 мин в помещении с температурой $21-24^{\circ} \mathrm{C}$. Перед исследованием пациенткам запрещали курение, прием пищи и напитков, влияющих на микроциркуляцию. Исследование проводили в одном и том же помещении в одно и то же время суток (обед с 12-13 ч; исследование с 15 до 17 ч), вне менструации. Пациентка находилась в положении сидя, верхняя конечность в легком сгибании размещена на столе, датчик устанавливали в области эпонихия 3-го пальца правой руки, при этом рука находилась на уровне сердца. При ОП манжету тонометра фиксировали на плече правой руки. Пробу проводили по следующей схеме: в течение одной минуты осуществляли регистрацию исходного уровня кровотока, запись останавливали. Затем создавали окклюзию путем быстрого нагнетания давления в манжете до уровня 230-250 мм рт. ст. и проводили дальнейшую регистрацию потока крови в течение 3 мин периода окклюзии. По истечении трехминутной окклюзии воздух из манжеты быстро выпускали, в течение последующих 6 мин регистрировали реакцию перфузии в ходе восстановления кровотока [11].

Данные, полученные при исследовании, обрабатывали с помощью программного обеспечения, входящего в комплект к используемому оборудованию. Оценивали следующие показатели: М - среднее арифметическое значение показателя микроциркуляции, измеряемое в перфузионных единицах (пф. ед.); $\sigma$ - среднее квадратичное отклонение (СКО) амплитуды колебаний кровотока от среднего арифметического значения М; с помощью вейвлет-преобразования показатели UVLF, VLF, LF, HF, CF - колебания кровотока соответственно эндотелиального, нейрогенного, миогенного, дыхательного, сердечного частотных диапазонов (пф. ед.); PKК - резерв капиллярного кровотока; угол $\alpha-$ угол подъема кривой реактивной гиперемии; I - индекс удельного потребления кислорода в ткани; Rc - внутрисосудистое сопротивление.

Статистическую обработку полученных данных проводили с использованием программы SPSS 21 (IBM SPSS, США). Описательные статистики для количественных показателей представляли в виде среднего значения М и стандартного отклонения $\mathrm{M} \pm \mathrm{CKO}$, либо в случае скошенной формы распределения - в виде медиан и межквартильного размаха. Для сравнения групп применяли однофакторный дисперсионный анализ (ANOVA) либо его непараметрический аналог - анализ Краскела-Уоллиса с последующим межгрупповым сравнением по критерию Манна-Уитни-Уилкоксона с поправкой Бонферрони. Для сравнения номинальных признаков применяли критерий $\chi^{2}$ Пирсона. Для оценки вклада отдельных признаков в риск развития тяжелой формы ПЭС применяли одномерную и многомерную логистическую регрессию. Качество прогнозирования оценивали с помощью ROC-анализа. Статистически значимыми результаты считали при $p<0,05$.

\section{РЕЗУЛЬТАТЫ ИССЛЕДОВАНИЯ}

Статистически значимых различий в клинико-диагностическом анамнезе, жалобах, данных обследования, размерах миомы матки ( ANovA $\left._{\text {AN }}=0,607\right)$, размерах доминантного узла $\left(p_{\text {ANovA }}=0,897\right)$ в исследуемых группах до ЭМА не выявлено. По локализации и количеству миоматозных узлов имелись различия (табл. 1).

Установлено, что у женщин с исходно низким кожным микрокровотоком М (7,90 пф. ед.) и высокой вариабельностью перфузии СКО (3,35 пф. ед.) после эмболизации развивается тяжелая степень ПЭС (табл. 2). Амплитудно-частотный спектр колебаний кровотока в покое не имеет диагностического значения, а при проведении ОП эндотелиальные и нейрогенные составляющие (активные механизмы микроциркуляции, связанные с притоком крови) могут быть предикторами тяжелого ПЭС. В частности, UVLF-колебания в ОП

Таблица. 1. Локализация и размеры доминантного узла в обследуемых группах

\begin{tabular}{|c|c|c|c|c|c|c|c|}
\hline \multirow{2}{*}{$\begin{array}{c}\text { Классификация миомы матки } \\
\text { по FIGO, } 2011 \text { г. }\end{array}$} & \multicolumn{3}{|c|}{ Степень ПЭС } & \multicolumn{3}{|c|}{ Межгрупповые сравнения } & \multirow{2}{*}{$\begin{array}{l}\text { Сравнение по } \\
\text { таблице в целом, } p\end{array}$} \\
\hline & $\begin{array}{c}\text { Легкая }(n=36) \\
\text { Абс. }(\%)\end{array}$ & $\begin{array}{c}\text { Средняя }(n=30) \\
\text { Абс. }(\%)\end{array}$ & $\begin{array}{c}\text { Тяжелая }(n=15) \\
\text { Абс. (\%) }\end{array}$ & $p^{1-2}$ & $p^{1-3}$ & $p^{2-3}$ & \\
\hline $\begin{array}{l}\text { Интрамуральный узел, в полость } \\
\text { матки до } 50 \% \text { (тип 2) }\end{array}$ & $16(44,4 \%)$ & $8(26,7 \%)$ & $6(40,0 \%)$ & 0,216 & 0,985 & 0,569 & \multirow{4}{*}{0,020} \\
\hline Интрамуральная миома (тип 4) & $16(44,4 \%)$ & $11(36,7 \%)$ & $1(6,7 \%)$ & 0,698 & 0,023 & 0,074 & \\
\hline $\begin{array}{l}\text { Субсерозно-мышечная миома, } \\
\text { до 50\% в брюшную полость (тип 5) }\end{array}$ & $3(8,3 \%)$ & $6(20,0 \%)$ & $6(40,0 \%)$ & 0,310 & 0,021 & 0,283 & \\
\hline Перешеечный узел (тип 8) & $1(2,8 \%)$ & $5(16,7 \%)$ & $2(13,3 \%)$ & 0,127 & 0,420 & 0,885 & \\
\hline Количество узлов & $1,53 \pm 1,02$ & $2,15 \pm 1,50$ & $2,62 \pm 1,75$ & 0,349 & 0,010 & 0,624 & 0,042 \\
\hline $\begin{array}{l}\text { Размеры доминантного узла } \\
\text { до ЭМА, мм }\end{array}$ & $58,32 \pm 17,30$ & $55,60 \pm 22,10$ & $54,76 \pm 17,70$ & 0,963 & 0,843 & 0,998 & 0,791 \\
\hline
\end{tabular}

Примечание: сравнение локализации миоматозных узлов выполнено по критерию $\chi^{2}$ Пирсона $\left(\chi^{2}=15,1\right)$, количества и размера узлов - с помощью однофакторного дисперсионного анализа. 
(эндотелиальные) в III группе исследуемых составили 1,76 пф. ед., что значительно ниже значений I группы (3,15 пф. ед. $\left.p_{1-3}<0,001\right)$ и II группы (2,76 пф. ед.; $\left.p_{2-3}=0,002\right) ;$ и VLFколебания в ОП (нейрогенные) в группе с тяжелым ПЭС ниже, чем в группах с легким $\left(p_{1-3}=0,001\right)$ и средним $\left(p_{2-3}=\right.$ 0,006) течением ПЭС.

Выявленные статистически значимые различия в антропометрических характеристиках (возраст, ИМТ), а также показателях кожного микрокровотока (М в покое, CKO в покое, PKК в ОП, угол $\alpha$ в ОП, UVLF в OП, VLF в ОП, Rc в ОП) до вмешательства послужили основой для выявления наиболее существенных факторов риска и предикторов (см. табл. 2). Математическим инструментом для оценки степени влияния изучаемых протективных факторов на ПЭС была логистическая регрессия. Прогнозирование риска развития тяжелой формы ПЭС по сравнению с легкой или средней степенями тяжести осуществляли, не разделяя последние две категории. На первом этапе были построены одномерные модели для основных показателей кровотока методом принудительного включения предикторов (табл. 3).

В ходе проведенного исследования нам удалось выделить ряд прогностических критериев развития тяжелого течения ПЭС в раннем послеоперационном периоде после проведения ЭМА. Среди антропометрических признаков статистически значимыми фракторами, влияющими на течение ПЭС, оказались возраст женщины и ИМТ. У обоих показателей отношения шансов меньше единицы: для возраста ОШ = 0,87 (0,79-0,96), а для ИМТ ОШ = 0,77
(0,63-0,94), что позволяет характеризовать их как протективные факторы. Согласно построенным математическим моделям, чем больше возраст и чем больше ИМТ пациентки, тем меньше у нее риск ПЭС. Для того чтобы выяснить, какие именно пороговые значения изучаемых признаков наилучшим образом прогнозируют развитие ПЭС, а также для количественной оценки предиктивных способностей каждого из изученных признаков были построены ROC-кривые (рис. 1), проанализированы их координаты и соответствующие значения чувствительности и специфичности. Установлено, что возраст женщины 38,5 лет и младше является фактором риска тяжелой ПЭС с чувствительностью 80\% и специсичностью 71\%. Следующим фрактором риска развития тяжелого ПЭС является ИМТ. Согласно полученным данным, женщины с нормальной массой тела и с десицитом массы тела оказываются в группе риска по тяжелому течению ПЭС. При пороговом значении ИМТ, равном 25 кг/м², чувствительность прогнозирования тяжелого ПЭС составила 80\%, специфичность - 62\%. Площадь под графиком характеристической кривой для возраста составила 0,76 0,068 ( $p=0,002)$, для ИМТ $0,74 \pm 0,073(p=0,003)$.

Из особенностей кровотока в покое высокоинформативным оказался показатель микроциркуляции $\mathrm{M}$, площадь под ROC-кривой для которого составила 0,81 \pm 0,05 (p < 0,001). Анализ координат ROC-кривой для М показал, что хорошей пороговой точкой является значение 9,55 пф. ед. У женщин с показателем перфузии в покое до

Таблица. 2. Антропометрические данные пациенток и параметры микроциркуляции в покое и при окклюзионной пробе до проведения ЭмА по группам ПЭС

\begin{tabular}{|c|c|c|c|c|c|c|c|}
\hline & $\begin{array}{l}\text { ПЭС легкая степень } \\
\text { (до } 7 \text { баллов) }\end{array}$ & $\begin{array}{c}\text { ПЭС средняя степень } \\
\text { (8-14 баллов) }\end{array}$ & $\begin{array}{c}\text { ПЭС тяжелая степень } \\
\text { (15-21 балл) }\end{array}$ & $p^{1-2}$ & $p^{1-3}$ & $p^{2-3}$ & $p^{\text {ANOVA }}$ \\
\hline Возраст, лет* & $39,72 \pm 6,47$ & $42,10 \pm 5,70$ & $35,07 \pm 5,74$ & 0,310 & 0,048 & 0,002 & 0,002 \\
\hline ИМТ, $\mathrm{Kг} / \mathrm{M}^{2 *}$ & $26,24 \pm 3,41$ & $27,86 \pm 6,39$ & $23,34 \pm 3,14$ & 0,515 & 0,020 & 0,008 & 0,013 \\
\hline \multicolumn{8}{|c|}{$\begin{array}{l}\text { Показатели микроциркуляции } \\
\text { в покое без проб }\end{array}$} \\
\hline М, пф. ед. & $22,33(12,98-24,03)$ & $17,55(10,38-19,15)$ & $7,90(5,40-15,60)$ & 0,110 & 0,002 & 0,011 & 0,003 \\
\hline$\sigma($ СКО), пф. ед. & $1,70(1,40-3,68)$ & $1,25(1,10-1,40)$ & $3,35(1,50-3,98)$ & $<0,001$ & 0,278 & $<0,001$ & $<0,001$ \\
\hline VLF, пф. ед. & $0,98(0,57-1,02)$ & $0,58(0,31-0,74)$ & $0,62(0,52-0,74)$ & $<0,001$ & 0,005 & 0,268 & $<0,001$ \\
\hline LF, пф. ед. & $0,54(0,45-0,81)$ & $0,42(0,28-0,69)$ & $0,42(0,38-0,54)$ & 0,037 & 0,054 & 0,791 & 0,053 \\
\hline HF, пф. ед. & $0,17(0,14-0,19)$ & $0,16(0,13-0,22)$ & $0,16(0,14-0,17)$ & 0,583 & 0,448 & 0,837 & 0,729 \\
\hline CF, пф. ед. & $0,15(0,14-0,24)$ & $0,22(0,13-0,37)$ & $0,17(0,14-0,26)$ & 0,017 & 0,279 & 0,242 & 0,047 \\
\hline 1 & $9,45(4,67-12,25)$ & $10,93(8,96-19,47)$ & $3,88(2,61-4,88)$ & 0,017 & $<0,001$ & $<0,001$ & $<0,001$ \\
\hline $\mathrm{Rc}$ & $0,19(0,10-0,27)$ & $0,38(0,24-0,47)$ & $0,18(0,08-0,21)$ & $<0,001$ & 0,489 & $<0,001$ & $<0,001$ \\
\hline \multicolumn{8}{|c|}{$\begin{array}{l}\text { Показатели кровотока } \\
\text { в окклюзионной пробе }\end{array}$} \\
\hline М, пф. ед. & $15,00(6,95-17,03)$ & $12,55(9,05-21,98)$ & $14,80(3,60-17,50)$ & 0,567 & 0,166 & 0,097 & 0,220 \\
\hline$\delta(\mathrm{CKO})$ & $11,40(7,55-13,63)$ & $10,25(7,73-12,25)$ & $8,30(2,60-9,40)$ & 0,463 & 0,042 & 0,089 & 0,097 \\
\hline PKK, \% & $166,21(139,95-219,05)$ & $136,31(128,33-174,04)$ & $220,09(134,40-245,11)$ & 0,008 & 0,414 & 0,013 & 0,008 \\
\hline Угол $\alpha$ & $65,89(54,19-69,27)$ & $50,22(48,35-65,73)$ & $41,11(34,92-48,22)$ & 0,051 & $<0,001$ & $<0,001$ & $<0,001$ \\
\hline UVLF, пф. ед. & $3,15(2,63-3,72)$ & $2,76(2,29-3,74)$ & $1,76(1,21-2,65)$ & 0,241 & $<0,001$ & 0,002 & $<0,001$ \\
\hline VLF & $1,75(1,46-2,61)$ & $1,75(1,23-2,41)$ & $1,33(0,81-1,64)$ & 0,312 & 0,001 & 0,006 & 0,003 \\
\hline LF & $0,78(0,63-1,13)$ & $0,95(0,46-1,01)$ & $1,00(0,32-1,09)$ & 0,279 & 0,282 & 0,981 & 0,432 \\
\hline $\mathrm{HF}$ & $0,23(0,13-0,32)$ & $0,23(0,17-0,32)$ & $0,27(0,12-0,44)$ & 0,588 & 0,694 & 0,923 & 0,843 \\
\hline $\mathrm{CF}$ & $0,15(0,13-0,19)$ & $0,16(0,11-0,24)$ & $0,16(0,12-0,23)$ & 0,380 & 0,413 & 0,904 & 0,592 \\
\hline 1 & $5,90(4,33-7,28)$ & $7,77(4,68-8,42)$ & $4,68(1,82-9,20)$ & 0,039 & 0,942 & 0,253 & 0,125 \\
\hline $\mathrm{Rc}$ & $0,04(0,03-0,04)$ & $0,04(0,03-0,07)$ & $0,06(0,05-0,09)$ & 0,124 & $<0,001$ & 0,004 & $<0,001$ \\
\hline
\end{tabular}

Примечание: * — данные представлены в виде $\mathrm{M} \pm \mathrm{CKO}$, в остальных случаях — в виде медианы и межквартильного размаха. 
планируемого вмешательства ниже 9,55 пф. ед. можно ожидать тяжелую форму ПЭС с чувствительностью 73\% и специфичностью 83\%. Выделение отдельных колебаний в микроциркуляторном русле с помощью вейвлетанализа в состоянии покоя без дополнительных проб прогностической ценности для диагностики тяжелого ПЭС не имело.

Проведение окклюзионной пробы, позволяющее моделировать ответ организма на острую гипоксию, показало высокий прогностический потенциал угла восстановления $\alpha$ (площадь под ROC-кривой 0,87 \pm 0,059; $p<0,001$ ) (рис. 1Г). Быстрое восстановление после трехминутной окклюзии уменьшает риск тяжелого ПЭС (ОШ = 0,85 (0,78-0,93); $p<0,001)$. Оптимальной пороговой точкой, на наш взгляд, является значение $\alpha$, равное 48 , при котором чувствительность и специфичность прогноза равны 80\%.

На следующем этапе были разработаны многомерные прогностические модели логистической регрессии с помощью пошагового отбора потенциальных предикторов (табл. 4). Поскольку различные показатели кровотока получаются из обработки одного сигнала, а некоторые являются производным от первичных показателей, все они в той или иной степени взаимосвязаны. Поэтому предложено несколько моделей сопоставимого качества (рис. 2).

Модель 1 построена по антропометрическим данным. Качество прогнозирования при пороговой вероятности 0,25: чувствительность - 67\%, специфичность - 88\%. Оба признака имеют ОШ меньше единицы, следовательно, их высокие значения являются протективными факторами; AUC $-0,81 \pm 0,058(p<0,001)$.

Модель 2 построена по антропометрическим данным и по состоянию кровотока в покое. В результате пошагового отбора в нее были включены только возраст и М. Качество прогнозирования при пороговой вероятности 0,35: чувствительность - 73\%, специфичность - 89\%; AUC $0,89 \pm 0,04(p<0,001)$.

Модель 3 построена по антропометрическим данным и неспектральным показателям ОП (углу а и РКК). Угол а наиболее сильно влияет на исход признаков: его снижение ассоциировано с повышением риска тяжелой формы ПЭС. Качество прогнозирования при пороговой вероятности 0,25: чувствительность - 87\%, специфичность - 91\%; AUC $-0,93 \pm 0,042(p<0,001)$.

Модель 4 близка по сути к модели 3. Для ее создания в качестве потенциальных предикторов использованы спектральные показатели ЛДФ в ОП по результатам вейвлет-анализа исходного сигнала. Согласно данной модели, наиболее существенным протективным фактором являются эндотелиальные колебания: их высокие значения снижают риск тяжелого формы ПЭС: ОШ - 0,09 (95\% ДИ: 0,02-0,47), что хорошо согласуется с выявленным ранее аналогичным эффектом угла $\alpha$, который также отражает интенсивность восстановления кровотока после ОП. Повышают риск тяжелого ПЭС высокие значения I и Rc. Качество прогнозирования при пороговой вероятности 0,25: чувствительность - 87\%, специфичность - 98\%; AUC $-0,94 \pm 0,056(p<0,001)$.

Таблица. 3. Оценка прогностических способностей антропометрических показателей и особенности микроциркуляции для прогнозирования тяжелого ПЭС по данным одномерных логистических регрессий

\begin{tabular}{|c|c|c|}
\hline Предиктор в модели & ОШ (95\% ди) & $p$ \\
\hline Антропометрические показатели & - & - \\
\hline Возраст, лет & $0,87(0,79-0,96)$ & 0,005 \\
\hline ИМТ, $\mathrm{Kr} / \mathrm{M}^{2}$ & $0,77(0,63-0,94)$ & 0,010 \\
\hline Показатели микроциркуляции в покое & - & - \\
\hline М, пф.ед. & $0,83(0,74-0,92)$ & 0,001 \\
\hline $\mathrm{CKO}, \delta$ & $1,70(1,09-2,64)$ & 0,019 \\
\hline VLF & $0,38(0,05-3,20)$ & 0,375 \\
\hline LF & $0,23(0,02-3,16)$ & 0,271 \\
\hline $\mathrm{HF}(\text { шаг } 0,1)^{*}$ & $0,50(0,15-1,63)$ & 0,248 \\
\hline $\mathrm{CF}$ & $0,15(0,00-20,14)$ & 0,452 \\
\hline 1 & $0,58(0,42-0,80)$ & 0,001 \\
\hline $\mathrm{Rc}(\text { шаг } 0,1)^{*}$ & $0,58(0,35-0,96)$ & 0,033 \\
\hline Показатели микроциркуляции в окклюзионной пробе & - & - \\
\hline М, пф. ед. & $0,94(0,86-1,03)$ & 0,166 \\
\hline CKO, $\delta$ & $0,82(0,71-0,96)$ & 0,014 \\
\hline PKK, \% & $1,02(1,00-1,03)$ & 0,008 \\
\hline Угол $\alpha$ & $0,85(0,78-0,93)$ & $<0,001$ \\
\hline UVLF & $0,23(0,10-0,53)$ & 0,001 \\
\hline VLF & $0,14(0,04-0,53)$ & 0,004 \\
\hline LF & $0,46(0,07-3,18)$ & 0,430 \\
\hline HF (шаг 0,1)* & $1,41(0,85-2,32)$ & 0,179 \\
\hline $\mathrm{CF}$ & $0,87(0,00-183,07)$ & 0,959 \\
\hline I & $1,07(0,91-1,26)$ & 0,422 \\
\hline $\mathrm{Rc}^{\star \star}($ шаг 0,01$)$ & $1,68(1,27-2,21)$ & $<0,001$ \\
\hline
\end{tabular}

Примечание: * - отношение шансов рассчитано на увеличение данного предиктора на 0,1; ** — отношение шансов рассчитано на увеличение данного предиктора на 0,01. 


\section{ОБСУЖДЕНИЕ РЕЗУЛЬТАТОВ}

По результатам других авторов, ПЭС тяжелой степени развивается у 10,2\% пациенток, перенесших ЭМА [3], по нашим данным - у 18,52\%, что может быть связано с тем, что у нас было больше критериев исключения в связи с проведением ЛДФ, т. е. заболевания препятствующие (искажающие) исследованию микрокровотока в ногтевом ложе. Известно, что реакция на один и тот же раздражитель выражена у людей молодого возраста больше, чем у представителей старшего поколения [12], о чем, например, свидетельствует самооценка боли по шкале ВАШ. Возможно этим объясняется, что в группе с тяжелым течением ПЭС средний возраст пациенток был меньше (35,07 \pm 5,74 года), что отнесли к факторам риска $(p=0,005)$. ИМТ менее $25 \mathrm{k} г / \mathrm{M}^{2}$ также являлся фактором риска развития тяжелого ПЭС. По данным нашего исследования, при избыточном весе микроциркуляция выше, что способствует более легкому течению ПЭС, а все системы организма работают в повышенном регуляторном и компенсаторном режиме, в связи с чем метаболическая нагрузка, возникающая после ЭМА, переносится легче [12]. По другим данным, у 2,8\% пациенток с размером миомы матки до 12 недель и у 7,3\% с миомой более 12 недель после ЭМА возникал стойкий парез кишечника с рвотой, что связывают с рефлекторным действием ишемизированных участков матки на близлежащие интимно расположенные органы или транзиторной ишемией кишечника в результате случайной частичной эмболизации верхней брыжеечной артерии [3]. В проведенном нами исследовании средние размеры доминантного узла были небольшими и статистически не различались в группах (см. табл. 1) в силу дизайна исследования, хотя по данным исследования размеры

A

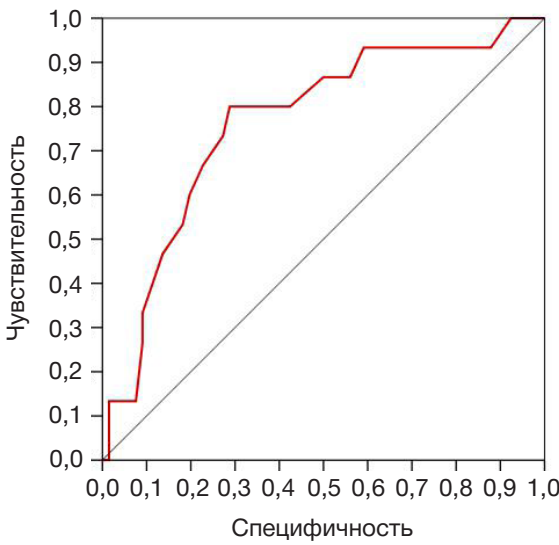

B

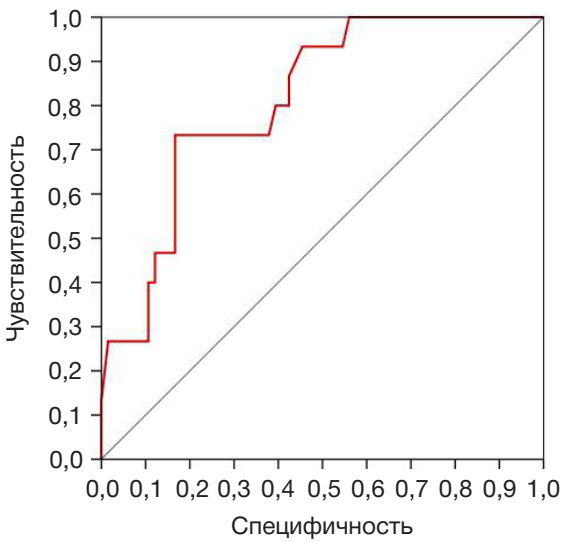

узла коррелируют с выраженностью болевого синдрома [13]. Мы отметили, что расположение миоматозных узлов влияет на течение послеоперационного периода. Фактором риска развития стало преобладание субсерозно-мышечной локализации узлов в группе пациенток с тяжелым течением ПЭС, так как ишемизированные узлы раздражают петли кишечника, приводят к тошноте, вздутию живота и другим признакам желудочно-кишечных нарушений после ЭМА, тем самым утяжеляя течение ПЭС в раннем послеоперационном периоде (см. табл. 1). К увеличению количества и длительности кровянистых выделений (один из симптомов ПЭС) приводят миомы матки с субмукозно-интерстициальным расположением узлов, которые преобладали в III группе [14].

У женщин с показателем перфузии в покое до планируемого вмешательства ниже 9,55 пф. ед. чаще развивается тяжелая форма ПЭС. Более низким был показатель М у большинства женщин с тяжелой формой ПЭС в последующем по сравнению с легкой: 53,3\% против $11,1 \%(p=0,004)$ и 23,3\% ( $p=0,094)$. У других исследователей, аналогично нашим данным, низкий показатель М тоже стал фактором риска развития послеоперационных осложнений, в сравнении с группой с высоким М [15]. Этим можно объяснить тот факт, что у пациенток со сниженным М для сохранения нормальной функции микроциркуляции в условиях изменения метаболических потребностей организма при развитии тяжелой степени ПЭС происходит увеличение микроциркуляции и ее регуляции (для этого необходимо время). Можно предположить, что у пациенток с высоким М вероятность развития тяжелого ПЭС ниже, так как микроциркуляция во всем организме в целом уже хорошая и не требуется время для подключения механизмов регуляции для поддержания гомеостаза $[12,16]$. Колебания кровотока UVLF, VLF, LF, HF, CF (табл. 3) прогностического

Б

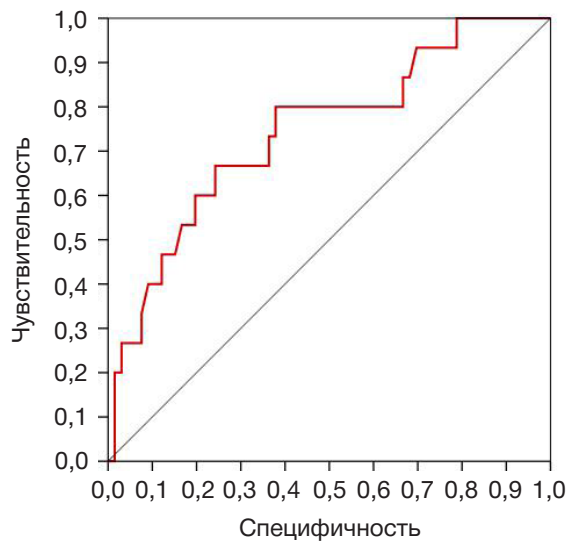

$\Gamma$

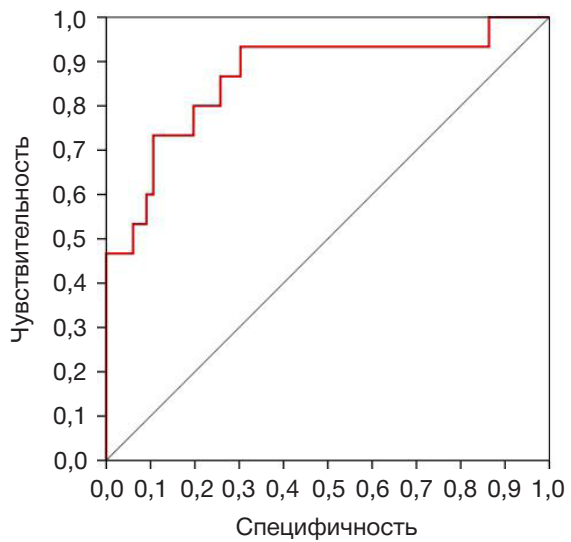

Pиc. 1. ROC-кривые прогностической способности возраста (A) и ИМТ (Б), показателя перфузии в состоянии покоя (В), угла восстановления кровотока $\alpha$ (Г) 
A

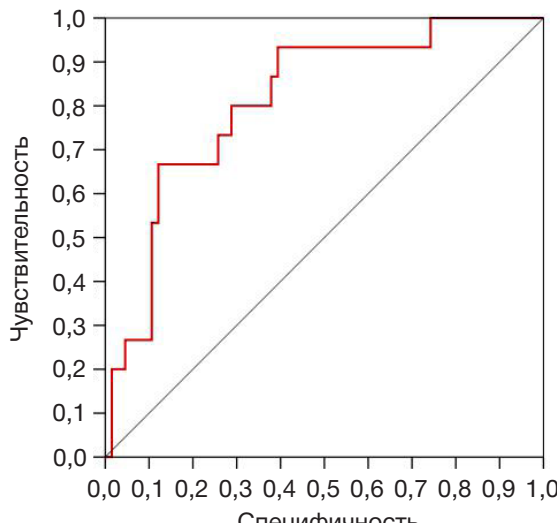

B

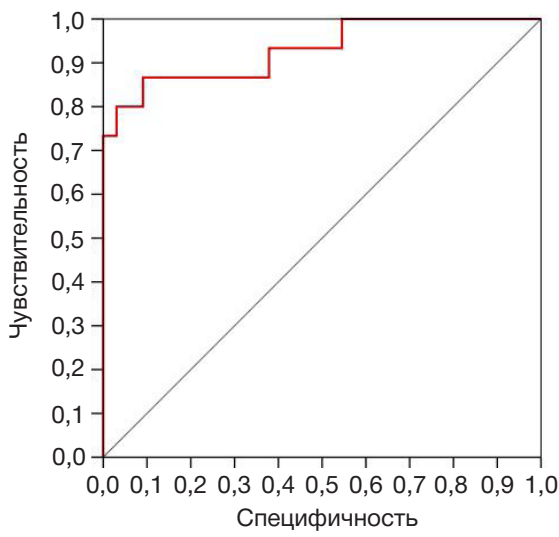

Б

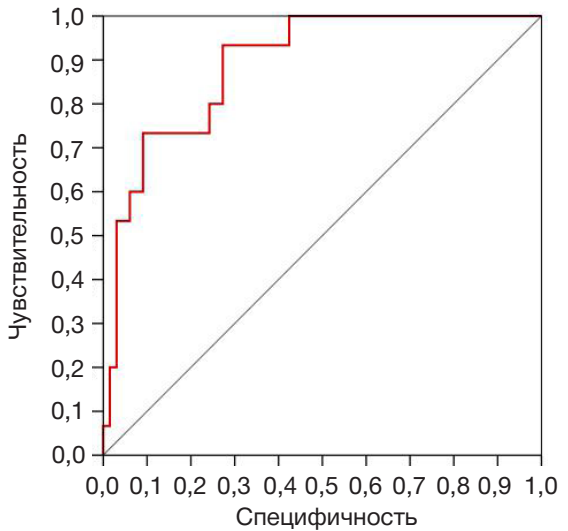

$\Gamma$

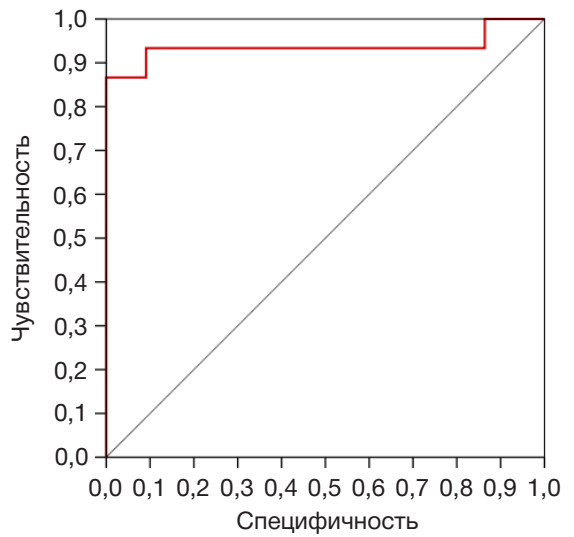

Рис. 2. ROC-кривые прогностической способности многомерных моделей 1 (А), 2 (Б), 3 (В), 4 (Г)

значения в покое не имели, при проведении ОП были выявлены изменения в эндотелиальных и нейрогенных колебаниях кровотока [17]. Так как ОП отражает интенсивность восстановления кровотока и резервные возможности микроциркуляторного русла, оцениваемые показатели обладают хорошей прогностической способностью. После трехминутного прекращения кровотока при декомпрессии кровоток в артерии восстанавливается и развивается реактивная гиперемия (РКK, угол $\alpha$, индекс потребления кислорода), которая через нейрогенную реакцию приводит к стимуляции выработки эндотелием оксида азота, вызывающего вазодилатацию $[11,16]$. Медленное восстановление микрокровотока является фактором риска развития тяжелого ПЭС. На основании полученных показателей были сформированы многомерные прогностические модели.

Таблица. 4. Оценка возможности использования антропометрических показателей и особенности микроциркуляции для прогнозирования тяжелого ПЭС по данным многомерных логистических регрессий

\begin{tabular}{|c|c|c|c|c|c|}
\hline Переменные в модели & Коэффициент регрессии, $b$ & SE $b$ & Статистика Вальда & ОШ (95\% дИ) & $p$ \\
\hline \multicolumn{6}{|l|}{ Модель 1} \\
\hline ИМТ, $\mathrm{k} \Gamma / \mathrm{M}^{2}$ & $-0,19$ & 0,1 & 3,83 & $0,83(0,69-1,00)$ & 0,05 \\
\hline Возраст, лет & $-0,11$ & 0,05 & 4,44 & $0,89(0,81-0,99)$ & 0,035 \\
\hline Константа & 7,49 & 2,68 & 7,82 & - & 0,005 \\
\hline \multicolumn{6}{|l|}{ Модель 2} \\
\hline М в покое, канал ПМ & $-0,23$ & 0,07 & 11,04 & $0,80(0,70-0,91)$ & 0,001 \\
\hline Возраст, лет & $-0,17$ & 0,06 & 8,17 & $0,84(0,75-0,95)$ & 0,004 \\
\hline Константа & 7,71 & 2,53 & 9,28 & - & 0,002 \\
\hline \multicolumn{6}{|l|}{ Модель 3} \\
\hline Угол $\alpha$ в ОП & $-0,19$ & 0,06 & 9,02 & $0,82(0,73-0,94)$ & 0,003 \\
\hline PKК в ОП, \% & 0,03 & 0,01 & 5,62 & $1,03(1,00-1,05)$ & 0,018 \\
\hline Возраст, лет & $-0,16$ & 0,08 & 4,41 & $0,85(0,74-0,99)$ & 0,036 \\
\hline Константа & 9,42 & 3,91 & 5,81 & - & 0,016 \\
\hline \multicolumn{6}{|l|}{ Модель 4} \\
\hline UVLF в ОП, пф. ед. & $-2,35$ & 0,81 & 8,42 & $0,09(0,02-0,47)$ & 0,004 \\
\hline І в ОП & 0,56 & 0,21 & 6,81 & $1,74(1,15-2,64)$ & 0,009 \\
\hline $\mathrm{Rc} \mathrm{в} \mathrm{O \Pi ^{* }}$ & 62,64 & 23,22 & 7,28 & $1,87(1,19-2,95)$ & 0,007 \\
\hline Возраст, лет & $-0,18$ & 0,08 & 5,12 & $0,83(0,71-0,98)$ & 0,024 \\
\hline Константа & 3,91 & 3,7 & 1,12 & - & 0,290 \\
\hline
\end{tabular}




\section{ВЫВОДЬ}

В ходе исследования нам удалось сформировать четыре прогностические модели развития тяжелого ПЭС. В состав многомерных моделей входят факторы риска развития тяжелого ПЭС. Из антропометрических данных в модели включены возраст пациенток младше 38,5 лет и ИМТ ниже 25 кг/M². Из показателей микроциркуляции, полученных с помощью ЛДФ, необходимо обратить внимание на фоновый уровень микроциркуляции; при М в покое менее 9,55 пф. ед. высок риск развития тяжелого ПЭС. Модели 1 и 2 с антропометрическими характеристиками и фоновым уровнем М имеют AUC в пределах, равных 0,8-0,9, что можно охарактеризовать как «очень хорошее». В модели 3 и 4 с AUC в пределах 0,9-1,0 («отличное») использованы показатели микроциркуляции при нагрузке (ОП) до ЭМА которые имеют хорошую прогностическую ценность. Чем выше в моделях значения I в ОП, Rс в ОП и PKK в ОП, тем выше риск развития тяжелой формы ПЭС. И наоборот, низкие показатели угла $\alpha$ в ОП и эндотелиальных колебаний UVLF в моделях ассоциированы с повышенным риском тяжелого ПЭС. Применяя эти прогностические модели в практической работе, врач может провести консультацию о возможном течении ПЭС и запланировать схему лечения в более ранний период после ЭМА, тем самым облегчив самочувствие пациенток в послеоперационном периоде.

\section{Литература}

1. Адамян Л. В., Серов В. Н., Сухих Г. Т., Филиппов О. С., редакторы. Клинические рекомендации. Акушерство и гинекология. Проблемы репродукции. Спец. вып. 2017; 23 (6).

2. Шаповалова А. И. Лейомиома матки и репродукция. Журнал акушерства и женских болезней. 2019; 68 (1): 93-101. https:// doi.org/10.17816/JOWD68193-101).

3. Доброхотова Ю. Э., редактор. Эмболизация маточных артерий. Спб: Экстен Медикал, 2013; 112 с.

4. Marín-Sánchez P, Sánchez-Ferrer ML. Conservative management of vesical-vaginal fistula after partial uterine and bladder necrosis due to embolization as a treatment for postpartum hemorrhage. Int Urogynecol J. 2015; 26 (5): 773-4. DOI: 10.1007/s00192014-2617-1.

5. Pillai AK, Kovoor JM, Reis SP, et al. Exposure of a Uterine Fibroid into the Small Bowl through Uteroenteric Fistula Presenting with Bowel Obstruction after Uterine Fibroid Embolization: Case Report with Histopathological Correlation. J Vasc Interv Radiol. 2016; 27 (5): 762-4. DOI: 10.1016/j. jvir.2015.11.047.

6. Yu Q, Gabriel G, Hoffman M, Sanampudi S, Jassim T, Raissi D. Uterine-sparing management of pyomyoma after uterine fibroid embolization. Radiol Case Rep. 2019 Jun 12; 14 (8): 1031-5. DOI: 10.1016/j.radcr.2019.05.009

7. Горюнова Т. В., Агапов В. К., Цвиркун В. В., ГолощаповАксенов Р. С., Скруберт В. С., Климов М. М., авторы; Горюнова Т. В., патентообладатель. Способ лечения миомы матки. Патент РФ №2289415, А61К35/14, 20.12.2006.

8. Пирогова В. И., Галанова З. М., Гарипов Р. М., Мухаметвалеева Г. Р, Галимов О. В., Бузаев И. В., и др., авторы; Башкирский государственный медицинский университет, петентообладатель. Способ просилактики послеоперационных осложнений эмболизации маточных артерий при миомах матки. Патент РФ №2364335, A61B5/1473, A61K49/12, 20.08.2009.

9. Юдина Т. А., Манухин И. Б., Тихомиров А. Л. Оптимизация постэмболизационного периода у больных миомой матки.

\section{References}

1. Adamyan LV, Serov VN, Sukhikh GT, Filippov OS, redactory. Klinicheskiye rekomendatsii. Akusherstvo i ginekologiya. Problemy reproduktsii. Spets vyp. 2017; 23 (6). Russian.

2. Shapovalova A. I. Lejomioma matki i reprodukciya. Zhurnal akusherstva i zhenskix boleznej. 2019; 68 (1): 93-101. https:// doi.org/10.17816/JOWD68193-101). Russian.

3. Dobrohotova YuE, redaktor. Embolizaciya matochnyh arterij. Spb: Eksten Medikal, 2013; 112 s. Russian.

4. Marín-Sánchez P, Sánchez-Ferrer ML. Conservative management of vesical-vaginal fistula after partial uterine and bladder necrosis due to embolization as a treatment for postpartum hemorrhage. Int Urogynecol J. 2015; 26 (5): 773-4. DOI: 10.1007/s00192-014-2617-1.

5. Pillai AK, Kovoor JM, Reis SP, et al. Exposure of a Uterine Fibroid

Акушерство и гинекология. 2017; (12): 110-4. DOI: https:// dx.doi.org/10.18565/aig.2017.12.110-114.

10. Дамиров М. М., Шахова О. Б., Саттарова З. И., Олейникова О. Н. Современные подходы к диагностике нарушений микроциркуляции в гинекологической практике (обзор литературы). Журнал им. Н. В. Склифосовского «Неотложная медицинская помощь». 2016; (1): 40-4.

11. Крупаткин А. И., Сидоров В. В. Лазерная доплеровская флоуметрия микроциркуляции крови: рук-во для врачей. М.: Медицина, 2005; 126 с.

12. Пальцев М. А., Пауков В. С., редакторы. Патология в 2-х томах: учебник. М.: ГЭОТАР-Медиа, 2010. 1024 с.

13. Сосин С. А., Приворотский В. В., Зазерская И. Е., Кустаров В. Н. Прогностические признаки выраженности болевого синдрома после эмболизации маточных артерий у женщин с лейомиомой матки. Гинекология. 2017; 19 (5): 30-3. DOI: 10.26442/2079-5696_19.5.30-33.

14. Нурмухаметова Э. Т., Шляпников М. Е. Архитектоника миоматозных узлов у женщин, поступивших на лечение методом эмболизации маточных артерий. Вестник медицинского института «Реавиз»: Реабилитация, врач и здоровье. 2019; 2 (38): 48-54.

15. Мусин И. И., Фаткуллина И. Б., Газизова Г. Х., Попова Е. М., Молоканова А. Р. Применение лазерной допплеровской флоуметрии и биологической обратной связи с целью профилактики эрозий сетчатого протеза. Практическая медицина. 2019; 17 (4): 88-91. DOI: 10.32000/2072-1757-2019-4-88-91.

16. Козлов В. И., Азизов Г. А., Гурова О. А., Литвин Ф. Б. Лазерная допплеровская флоуметрия в оценке состоянии и расстройств микроциркуляции крови. Методическое пособие РУДН. Москва. 2012, 32 с.

17. Нурмухаметова Э. Т., Шляпников М. Е. Диагностическая значимость оценки состояния периферического кровотока после эмболии маточных артерий. Практическая медицина. 2018; 16 (8): 106-10.

into the Small Bowl through Uteroenteric Fistula Presenting with Bowel Obstruction after Uterine Fibroid Embolization: Case Report with Histopathological Correlation. J Vasc Interv Radiol. 2016; 27 (5): 762-4. DOI: 10.1016/j. jvir.2015.11.047.

6. Yu Q, Gabriel G, Hoffman M, Sanampudi S, Jassim T, Raissi D. Uterine-sparing management of pyomyoma after uterine fibroid embolization. Radiol Case Rep. 2019 Jun 12; 14 (8): 1031-5. DOI: 10.1016/j.radcr.2019.05.009.

7. Goryunova TV, Agapov VK, Cvirkun VV, GoloshchapovAksenov RS, Skrubert VS, Klimov MM, avtory; Goryunova TV, patentoobladatel'. Sposob lecheniya miomy matki. Patent RF № 2289415, A61K35/14, 20.12.2006. Russian.

8. Pirogova VI, Galanova ZM, Garipov RM, Muhametvaleeva GR, 
Galimov OV, Buzaev IV, i dr, avtory; Bashkirskij gosudarstvenny] medicinskij universitet, petentoobladatel'. Sposob profilaktiki posleoperacionnyh oslozhnenij embolizacii matochnyh arterij pri miomah matki. Patent RF № 2364335, A61V5/1473, A61K49/12, 20.08.2009. Russian.

9. Yudina TA, Manuhin IB, Tihomirov AL. Optimizaciya postembolizacionnogo perioda $u$ bol'nyh miomoj matki. Akusherstvo i ginekologiya. 2017; (12): 110-4. DOI: https:// dx.doi.org/10.18565/aig.2017.12.110-114. Russian.

10. Damirov MM, Shaxova OB, Sattarova ZI, Olejnikova ON. Sovremenny'e podxody' $\mathrm{k}$ diagnostike narushenij mikrocirkulyacil $\checkmark$ ginekologicheskoj praktike (obzor literatury). Zhurnal im. N.V. Sklifosovskogo "Neotlozhnaya medicinskaya pomoshh». 2016; (1): 40-4. Russian.

11. Krupatkin Al, Sidorov W. Lazernaya doplerovskaya floumetriya mikrocirkulyacii krovi: ruk-vo dlya vrachej. M.: Medicine, 2005; 126 s. Russian.

12. Palcev MA, Paukov VS, redaktory. Patologiya $\vee 2-h$ tomah: uchebnik. M.: GEOTAR-Media, 2010; 1024 s. Russian.

13. Sosin SA, Privorotskij V, Zazerskaya IE, Kustarov VN. Prognosticheskie pri-znaki vy'razhennosti bolevogo sindroma posle èmbolizacii matochnỳx arterij u zhenshhin s lejomiomoj matki. Ginekologiya. 2017; 19 (5): 30-3. DOl: 10.26442/20795696_19.5.30-33. Russian.

14. Nurmukhametova ET, Shlyapnikov ME. Arkhitektonika miomatoznykh uzlov u zhenshchin. postupivshikh na lecheniye metodom embolizatsii matochnykh arteriy. Vestnik meditsinskogo instituta «Reaviz»: Reabilitatsiya, vrach i zdorovye. 2019; 2 (38): 48-54. Russian

15. Musin II, Fatkullina IB, Gazizova GH, Popova EM, Molokanova AR. Primenenie lazernoj dopplerovskoj floumetrii i biologicheskoj obratn oj svyazi s cel'yu profilaktiki erozij setchatogo proteza. Prakticheskaya medicina. 2019; 17 (4): 88-91. DOI: 10.32000/2072-1757-2019-4-88-91. Russian.

16. Kozlov VI, Azizov GA, Gurova OA, Litvin FB. Lazernaya dopplerovskaya floumetriya $\vee$ ocenke sostoyanii i rasstrojstv mikrocirkulyacii krovi. Metodicheskoe posobie RUDN. Moskva, 2012; 32 s. Russian.

17. Nurmukhametova ET, Shlyapnikov ME. Diagnosticheskaya znachimost ocenki sostoyaniya perifericheskogo krovotoka posle e`mbolii matochny’x arterij. Prakticheskaya medicina. 2018; 16 (8): 106-10. Russian 